\title{
MEDICAMENTO E O USO OFF LABEL
}

\section{DRUG AND OFF-LABEL USE}

\author{
PAULA, Cristiane da Silva'; MIGUEL, Obdúlio Gomes²; MIGUEL, Marilis Dallarmi² \\ 1 - Doutoranda do Programa de Pós-Graduação em Ciências Farmacêuticas - Universidade Federal do \\ Paraná \\ 2 - Professor (a) do Departamento de Farmácia - Programa de Pós-Graduação em Ciências Farma- \\ cêuticas - Universidade Federal do Paraná
}

\section{RESUMO:}

O uso off label é quando um medicamento é utilizado para uma indicação diferente daquela que foi autorizada pelo órgão regulatório de medicamentos em um país, e para as quais não existem bases científicas adequadas. È o uso para uma indicação terapêutica não descrita em bula podendo também estar relacionado ao uso do medicamento em uma faixa etária diferente da recomendada. Algumas publicações fazem referências às justificativas e consequências do uso off labe/ dos medicamentos, além de propostas para a regulamentação do uso

Palavras-chave: Off label. Medicamento.

\section{ABSTRACT:}

The off-label use is when a drug is prescribed for an indication other than those authorized by the Regulatory Agency of medicines in a country, and for which there is no adequate scientific basis. It is the use for a therapeutic indication not described in the package insert and may also be related to the use of the product for ages other than those that were recommended Some papers make reference to the justifications and consequences of off label use of drugs, as well as proposals for regulating the use.

Keywords: Off- label. Medicine.

\section{NTRODUÇÃO}

No Brasil um medicamento somente poderá ser comercializado se for registrado no Ministério da Saúde (MS). O controle é realizado pela Agência Nacional de Vigilância Sanitária (ANVISA), e o registro é concedido após comprovação científica de que é seguro e eficaz para o uso a que se propõe (BRASIL, 1976).

\section{USO OFF LABEL}

O termo "off label" vem sendo utilizado mundialmente para descrever a prática de uso do medicamento para uma condição adversa daquela descrita em bula (ANSEL; POPOVICH;ALLEN JR, 2000). 
um produto, ele aprova também um "label", que em português traduz-se por "rótulo" ou "etiqueta" específica para este produto. O rótulo aprovado é representado pela bula (ANSEL; POPOVICH; ALLEN JR, 2000) e demonstra todo o processo de desenvolvimento do fármaco com informações sobre farmacocinética, farmacodinâmica, eficácia, toxicidade, indicações aprovadas, descrição das reações adversas, instruções sobre dosagem, via de administração e população de pacientes que fará uso do medicamento (PAGE, 2004; KAIRUZ, 2007; DRESSER; FRADER, 2009).

Estudos realizados após a comercialização do medicamento podem ampliar ou restringir as indicações inicialmente aprovadas, entretanto, o profissional legalmente habilitado pode decidir utilizá-lo para uma indicação não prevista em bula baseado em estudos em andamento ou em situações onde acredite poder beneficiar o paciente (ANVISA, 2005). Este tipo de prescrição não é proibida por lei sendo de total responsabilidade de quem prescreve e pode, em algumas situações, ser caraterizada como erro ou apenas um uso correto ainda não aprovado (ANVISA, 2005).

De acordo com Dooley (2007) 57\% de novas indicações para medicamentos já aprovados, são realizadas na prática clínica. Diante deste fato, Sox (2009) esclarece que muitas vezes este fato ocorre porque os fabricantes não são motivados a assumir os enormes custos de ensaios para testar outros efeitos dos medicamentos que já estão sendo comercializados.

Alguns autores (WANNMACHER, 2007) reforçam que apesar da prescrição não ser ilegal, só deve ser utilizada quando fizer parte de uma pesquisa formal ou houver o consentimento esclarecido do paciente; quando existir comprovação de eficácia ou em situações onde os potenciais benefícios superem os riscos.

\section{USO OFF LABEL EM POPULAÇÕES ESPECIAIS}

O uso off label de medicamentos é frequente em populações especiais, como crianças e mulheres grávidas pelo fato de que os ensaios clínicos não são rotineiramente realizados nestas populações.

Poucos estudos são realizados em pacientes pediátricos, porque é necessário o consentimento informado e autorização dos pais ou tutores das crianças, o que muitas vezes dificulta os ensaios. Além disso, não existe incentivo financeiro para as empresas farmacêuticas realizarem ensaios com a população pediátrica, ou seja, as empresas não terão um retorno monetário com as vendas para cobrir todo o custo dos ensaios clínicos (DOOLEY, 2007).

Também, não ocorrem em mulheres grávidas, devido ao risco de possíveis efeitos teratogênicos ao feto, e desta forma faltam voluntárias. Exemplo da importância 
deste fato foi a tragédia da talidomida, que reforça a necessidade de se criar uma forma de avaliar a segurança do uso de medicamentos (DOOLEY, 2007).

GAZARIAN (2006) reporta em seu artigo que o uso off label de medicamentos é uma prática comum, com taxas de até $40 \%$ em adultos e $90 \%$ em pacientes pediátricos na Austrália. Um estudo constatou que nos Estados Unidos $21 \%$ dos medicamentos prescritos são utilizados para indicações não aprovadas pelo FDA. Entre os medicamentos com indicação off label, 73\% não possuíam evidências de eficácia clínica e apenas $27 \%$ apresentavam fortes evidências científicas. Em oncologia, onde geralmente encontram-se medicamentos de custos elevados com efeitos secundários graves, cinco dos medicamentos mais amplamente prescritos foram utilizados com indicações offlabelem $50 \%$ dos casos (GILLICK, 2009).

\section{JUSTIFICATIVAS E CONSEQUENCIAS DO USO OFF LABEL}

A justificativa ética para a ocorrência de prescrição off label é que ela pode proporcionar o melhor tratamento disponível para um determinado paciente. Isto contrasta com a justificativa ética para a realização de ensaios clínicos, que é o de desenvolver novas terapias ou esclarecer o melhor uso dos tratamentos existentes para futuros pacientes (DRESSER; FRADER, 2009).

Agravidade da patologia também pode ser uma justificativa para se prescrever e utilizar medicamentos não aprovados, tendo em vista a relação risco benefício (CARVALHO et al., 2003). Neste contexto, os prescritores são confrontados com o dilema de prescrever medicamentos sem informação suficiente para dar-lhes segurança, ou deixar seus pacientes sem terapia efetiva e, às vezes salvadora. Diante destes aspectos, de acordo com Carvalho et al. (2003) é fundamental basear-se na literatura médica, na experiência prévia ou na opinião de especialista que pode dar embasamento aos profissionais que prescrevem. De acordo com Carvalho et al. (2003), a orientação que acompanha os medicamentos (bula), embora seja importante fonte de informação não é fator determinante de prescrição adequada, e menos ainda um substituto do julgamento médico.

O uso off label também pode promover avanços dos conhecimentos médicos sobre o medicamento, principalmente no caso de patologias que não são tão frequentes na população ou raras onde os altos custos do processo de regulamentação não despertariam interesse do fabricante tendo em vista o pequeno mercado. Neste contexto, pode-se estar privando pacientes que sofrem destas patologias de tratamentos que podem trazer grandes benefícios. Pode-se incluir neste caso também pacientes não responsivo à terapia padrão ou sem alternativas de tratamento (TABARROK, 2009). Curtiss e Fairman (2009) defendem que a promoção 
do uso off label pode ser adequada nos casos onde um fármaco pode melhorar a qualidade de vida do paciente.

Gillick (2009) reporta algumas vantagens obtidas com o uso off label de medicamentos tais como a descoberta de novas indicações importantes com a flexibilização do uso; essencial para áreas como pediatria evitando o processo demorado e caro de se alterar as aprovações do registro de um medicamento.

Pode permitir inovação na prática clínica, particularmente quando o tratamento aprovado não foi eficaz, fornecer aos pacientes e médicos acesso precoce a medicamentos potencialmente valiosos e permitir aos médicos adotarem novas práticas baseadas em evidências emergentes, assim como pode ser a única opção disponível para certas condições (STAFFORD, 2008).

Também pode ser aprovado para uma condição e prescrito para outra condição na qual apresente tem similaridade genética ou fisiológica. Podem prescrever o medicamento para pacientes que apresentam um quadro mais grave ou menos grave da doença ou para situações que causem sintomas semelhantes. Aplicações off label podem surgir através de um processo de pesquisa ou "descoberta de campo", em que os médicos identificam novas aplicações (DRESSER; FRADER, 2009).

O uso off label pode ser suportado por diferentes níveis de evidências. Autoridades reconhecem uma hierarquia de evidências científicas e clínicas que podem justificar as intervenções médicas. Normalmente estão no topo os grandes estudos randomizados controlados, seguidos por pequenos estudos randomizados, estudos de coorte, estudos caso-controle, pouco controlados ou não controlados, relato de casos e opinião de especialistas (DRESSER; FRADER, 2009). Evidências de alta qualidade não só protegem o paciente de intervenções ineficazes e perigosas como também aumentam o acesso a tratamentos benéficos. Pesquisa de alta qualidade, indicando que uso off label é seguro e eficaz, pode também incentivar os fabricantes a obter a aprovação para novos usos (DRESSER; FRADER, 2009).

A falta de formas farmacêuticas adequadas faz com que ocorram adaptações que se não forem realizadas adequadamente podem trazer riscos de inexatidão de dose, contaminação durante a manipulação, perda de estabilidade, incompatibilidades, interações, diminuição da eficácia, a baixa adesão dos pacientes devido ao sabor e riscos para os trabalhadores de saúde (SOUZA; CABRAL; HUF, 2009). Além disso, no caso de comprimidos revestidos, e este revestimento tiver como função proteger a substância ativa da degradação devido à ação do suco gástrico, a sua pulverização não constitui uma opção tecnicamente correta (PINTO; BARBOSA, 2008). Uma formulação ideal deve ser preparada baseada em informações científicas que respaldem este preparo com determinação do prazo de validade. Entretanto, essa 
não é a realidade observada, que pela carência de apresentações adequadas, verificase mais frequentemente, a adaptação de formas farmacêuticas sólidas para líquidas e a prescrição de formulações magistrais (DA COSTA; LIMA; COELHO, 2009) sem bases científicas. Neste contexto, a falta de produtos com características adequadaspode ser um importante fator de risco para reações adversas à medicamentos e intoxicações (DA COSTA; REY, COELHO, 2009).

Deve-se ressaltar que quando houver necessidade de manipulação de medicamentos é fundamental a infraestrutura laboratorial adequada nas farmácias e profissionais qualificados, situação que de acordo com o autor ainda precisa ser melhorada no Brasil. Enfim, a importância de se incluir conhecimentos básicos em farmacotécnica e cálculos farmacêuticos no âmbito da educação a fim de auxiliar o prescritor na delicada tarefa de adaptar formulações e dosagens (DA COSTA; REY; COELHO, 2009).

O médico responsável pela prescrição deve antes de tudo avaliar se existem provas suficientes para justificar um uso off label, publicações e pesquisas quando estiverem faltando evidências adequadas, além de informar os pacientes sobre as incertezas e os custos potenciais associados com a prescrição off label. Além disso, as autoridades federais devem acompanhar sistematicamente as respostas do paciente aos usos off label, regularmente recolher e divulgar informações, e analisar as propostas para regular certos usos, bem como outras propostas de medidas políticas que podem diminuir os riscos e prescrições ineficazes (DRESSER; FRADER, 2009).

Ao contrário dos medicamentos prescritos para indicações aprovadas pelo FDA e ANVISA, falta um controle científico rigoroso quando utilizados de forma off label. Apesar de preocupações com a segurança do paciente e os custos para o sistema de saúde, pouco se sabe sobre a frequência de uso off label do medicamento ou o grau de evidência científica para apoiar essa prática (RADLEY; FINKELSTEIN; STAFFORD, 2006).

\section{PROPOSTAS DE REGULAÇÃO DO USO OFFLABEL}

Várias propostas de regulação do uso do uso off label são sugeridas por diversos autores, e nesta perspectiva alguns defendem uma autorização prévia, onde um órgão patrocinado pelo governo federal deve decidir quais indicações são justificadas (ANSANI et al., 2006).

No sistema sugerido por Gillick (2009), deve haver preocupação somente com medicamentos off label de alto custo ou potencialmente perigosos. De acordo com o autor não se justifica controlar todos os medicamentos que são utilizados com indicação diferente da recomendada, principalmente medicamentos com poucos 
efeitos colaterais e de baixo custo. Sugere-se que as prescrições de alto custo sejam acompanhadas por um formulário indicando a condição para a qual o medicamento está sendo prescrito; que exista intensificação do sistema de vigilância póscomercialização, e que sempre haja um termo de consentimento esclarecido para o paciente. Diante destas medidas, o autor esclarece que o paciente está amparado pelos benefícios de novas terapias.

Mossman (2009) discorda de alguns analistas que sugerem que a prescrição off labe/ seja equivalente à experimentação humana. Para o autor na pesquisa clínica o pesquisador investiga hipóteses para obter um conhecimento generalizado, ao passo que a terapia médica visa beneficiar pacientes individuais.

Wilkes (2008) reforça a importância do consentimento informado do paciente antes de se executar um teste, iniciar um tratamento especial ou tratamento que envolva incertezas. O médico deve desta forma divulgar a natureza da intervenção, prós e contras da intervenção, alternativas e prós e contras das alternativas, cumprindo desta forma com as obrigações éticas. Neste contexto é importante que o paciente participe do processo de tomada de decisões médicas em situações de incertezas que é essencial para respeitar a sua autonomia (WILKES, 2008).

Gazarian et al. (2006) publicaram orientações para ajudar os profissionais de saúde julgar a adequação de um tratamento off label. Suas recomendações são baseadas na avaliação das evidências que sustentam utilização indevida e na identificação de categorias de prescrição off label (rotina, uso no âmbito da investigação formal, e uso excepcional). Para divulgação de informações oportunas e precisas para os médicos, Levêque (2008) sugere a criação de um centro independente de informação sobre o fármaco, que acompanha os estudos off label e atualizar as informações sobre o medicamento.

De acordo com Gazarian et al. (2006) quando existem evidências de alta qualidade que apoiem o uso off label de um medicamento o processo usual de obtenção de consentimento (documentado) para o tratamento é recomendável. Isso inclui obter informações adicionais sobre as incertezas e discutir com o paciente/pais o motivo para a utilização do medicamento, terapias alternativas e possíveis reações adversas. Quando não existe evidência de alta qualidade apoiando o uso, porém seja necessário para um determinado medicamento, uma avaliação sobre os possíveis benefícios e os riscos devem ser bem compreendidas. Alternativamente, o uso pode ocorrer no contexto de uma proposta formal de investigação após avaliação e aprovação por uma comissão institucional de ética em pesquisa. Em ambos os casos, o consentimento informado é necessário (GAZARIAN et al. , 2006). 


\section{REFERÊNCIAS}

ANSANI, N.; SIRIO, C.; SMITHERMAN, T.; FEDUTES-HENDERSON, B.; SKLEDAR, S. WEBER, R.J. Designing a strategy to promote safe, innovative off-label use of medications. Am J Med Qual, United States, v. 21, n.4, p. 255-261, jul/aug. 2006.ANSEL, H.C.; POPOVICH, N.G.; ALLEN JR., L.V. Farmacotécnica. Formas Farmacêuticas e Sistema de Liberação de Fármacos. 6ª Ed. São Paulo, 2000.

ANVISA-AGÊNCIA NACIONAL DE VIGILÂNCIA SANITÁRIA. Medicamentos. Como a ANVISA vê o uso off label de medicamentos - 2005. Disponível em: < http://www.anvisa.gov.br/medicamentos/registro/registro_offlabel.htm>. Acesso em: 06/06/2008.

BRASIL. Lei n 6360, de 23 de setembro de 1976. Dispõe sobre a Vigilância Sanitária a que ficam sujeitos os medicamentos, as drogas, os insumos farmacêuticos e correlatos, cosméticos, saneantes e outros produtos, e dá outras providências. Diário Oficial [da] Republica Federativa do Brasil, Brasília, DF, 24 set. 1976. Disponível em: <http://e-legis.anvisa.gov.br/>. Acesso em: 06/06/2008.

CARVAlHO, P. R. A.; CARVAlHO, C. G.; ALIEVI, P. T.; MARTINBIANCHO, J.; TROTTA, E. A. Identificação de medicamentos "não apropriados para crianças" em prescrições de unidade de tratamento intensivo pediátrica. Jornal de Pediatria, Porto Alegre, v. 79, n. 5, p. 397-402, set./out. 2003.

CURTISS, F.R; FAIRMAN, K.A. Contradictory Actions on Off-label Use of Prescription Drugs? The FDA and CMS Versus the U.S. Justice Department. Journal of Managed Care Pharmacy. United States, v. 15, n. 2, p. 161-165, March 2009.

DA COSTA, P. Q; REY, L. C; COELHO, H. L. L. Lack of Drug Preparations for Use in Children in Brasil. J.Pediatr, Rio de Janeiro. v. 85, n. 3, p. 229-235, mar. 2009.

DA COSTA, P. Q.; LIMA, J. E. S.; COELHO, H. L. L. Prescrição e preparo de medicamentos sem formulação adequada para crianças: um estudo de base hospitalar. Braz. J. Pharm. Sci., São Paulo, v. 45, n. 1, p. 57-66, jan./mar. 2009.

DOOLEY, A. Off-Label Drug Use. Medicine \& Health. v. 90, n. 2, p.63-65, 2007.

DRESSER, R.; FRADER, J. Off-Label Prescribing: A Call for Heightened Professional 
and Government Oversight. J Law Med Ethics, Boston, v. 37, n. 3, p. 476-486, 2009.

GAZARIAN, M.; KELLY, M.; MCPHEE, J. R; GRAUDINS, L. V.; WARD, R. L; CAMPBELL, T. J. Off-label use of medicines: consensus recommendations for evaluating appropriateness. The Medical Journal of Australia. Austrália, v. 185, n. 10, p. 544-547, nov. 2006.

GILLICK, M. R. Controlling Off-label Medication Use. Annals of Internal Medicine. United States, v. 150, n. 5, p. 344-347, mar. 2009.

KAIRUZ, T. E; GARGIULO, D.; BUNT, C.; GARG, S. Quality, Safety and Efficacy in the 'Off-Label' Use of Medicines. Current Drug Safety, United Arab Emirates, v. 2, n. 1, p. 89-95, jan. 2007.

LEVÊQUE, D. Off-label use of anticancer drugs. The Lancet Oncology, v. 9, n. 11, p. 1102-1107, nov 2008.

MOSSMAN, D. Why off-label isn't off base. OBG Management, v. 21, n. 3, mar. 2009.

PAGE, C. Regulamentação do Uso das Drogas. In: Farmacologia Integrada. $2^{\mathrm{a}}$ ed. Barueri SP: Manole, 2004. p. 83-88.

PINTO, S.; BARBOSA, C. M. Medicamentos Manipulados em Pediatria. Estado Actual e Perspectivas Futuras. Arq. Med., Porto, v. 22, n. 2/3, p. 75-84, 2008.

RADLEY, D.C.; FINKELSTEIN, S.N.; STAFFORD, R.S. Off-label Prescribing Among Office-Based Physicians. Arch Intern Med. Chicago, v. 166, n. 9, p. 1021-1026, may 2006.

SOUZA, A. H. A.; CABRAL, L. M.; HUF, G. Current practices on the use of sildenafil citrate for pulmonary arterial hypertension in Brazilian hospitals. BMC Research Notes, v. 30, n. 2, 2009. Disponível em: <http://www.biomedcentral.com/content/pdf/ 1756-0500-2-30.pdf>. Acesso em: 22/03/2009.

SOX, H. C. Evaluating Off-label Uses of Anticancer: Time for a Change. Annals of Internal Medicine, United States, v. 150, n. 5, p. 353-354, mar. 2009.

STAFFORD, R. S. Regulating Off-Label Drug Use - Rethinking the Role of the FDA. 
WANNMACHER, L. Aética do medicamento: múltiplos cenários. Brasília, v. 4, n.

WILKES, M.; JOHNS, M. Informed Consent and Shared Decision-Making: A Requirement to Disclose to Patients off-label Prescriptions. PLOS Medicine, United States, v. 5, n. 11, p. 1553-1556, nov. 2008. 